\title{
Novel low-PAPR parallel FSOK transceiver design for MC-CDMA system over multipath fading channels
}

\author{
Juinn-Horng Deng ${ }^{*}$ and Jeng-Kuang Hwang
}

\begin{abstract}
A low peak-to-average power ratio (PAPR) transceiver using a new parallel frequency-shift orthogonal keying (FSOK) technique is proposed for the multiuser uplink multi-carrier CDMA (MC-CDMA) system over multipath fading channels. By employing the frequency modulated and multiplexed FSOK techniques to combat the multiuser and parallel substream interferences, respectively, the system retains a low-PAPR transmitted signal and a low-complexity equalizer without any matrix inversion. At the basestation, a multiuser receiver is derived, which involves parallel FSOK despreading, demapping, and maximum likelihood decision rule to acquire M-ary modulation gain and frequency diversity gain. For higher link quality, a multiple input single output FSOK uplink system can flexibly be configured. Simulation results are included to demonstrate that the proposed system achieves the low-PAPR property, space-frequency diversity, and M-ary modulation gain. Compared to the existing MC-CDMA and SC-FDMA systems, the proposed system exhibits significant performance superiority.
\end{abstract}

Keywords: multi-carrier CDMA (MC-CDMA), frequency-shift orthogonal keying (FSOK), peak-to-average power ratio (PAPR), multiple input multiple output (MIMO), SC-FDMA

\section{Introduction}

Currently, low peak-to-average power ratio (PAPR) modulation schemes are highly recommended for uplink broadband wireless communications. Single-carrier frequency division multiple access (SC-FDMA) techniques [1-3], e.g., interleaved, distributed, and localized SCFDMA, have been proposed to achieve the low-PAPR requirement. SC-FDMA systems with different subcarrier assignment schemes can preserve the orthogonality among users, which facilitates multiuser communications and combats multiple access interference (MAI). Further, a frequency-domain equalizer is adopted by the SC-FDMA receiver to mitigate the multipath interference (MPI) effect and obtain the frequency diversity gain. In particular, the localized and distributed SCFDMA is now considered as a promising candidate technique to support multiuser uplink in future 4G wireless communications (e.g., long-term evolution) $[4,5]$. However, to cope with the MAI and MPI effects,

\footnotetext{
* Correspondence: jh.deng@saturn.yzu.edu.tw

Department of Communication Engineering, Yuan Ze University, Chungli, Taoyuan 32003, Taiwan, ROC
}

each uplink user in the distributed or localized SCFDMA systems is assigned to utilize the partial spectrum. Such a constraint may in fact deteriorate the PAPR property, as Horlin et al. [6] have indicated that the localized SC-FDMA has a larger PAPR than the cyclic prefix (CP) CDMA system, and also obtains a better link performance than the latter. However, the localized SC-FDMA is limited to the acquisition of partial frequency diversity since it utilizes only partial frequency subcarriers [1].

Based on the above discussion, to simultaneously achieve multiuser detection and low-PAPR, as well as obtain frequency diversity gain to the greatest possible extent, we propose a novel parallel frequency-shift orthogonal keying (FSOK) technique for multi-carrier CDMA (MC-CDMA) systems. In the literature, conventional MC-CDMA systems, such as the Walsh-Hadamard (WH) MC-CDMA system, experience limited uplink performance because of the presence of MAI, since the orthogonality among the composite signatures of different users no longer holds in the presence of multipath channels $[7,8]$. To eliminate MAI interference, 
Adachi and Nakagawa [9] have recently proposed a new MC-CDMA system using a conventional spreading code with user-specific phase rotated spreading codes, which can achieve multiple access communications. To overcome the MPI and MAI problems, we continue the work started in $[10,11]$ and propose a novel extension called the parallel FSOK MC-CDMA system to support robust multiuser uplink communications over multipath channels while preserving the low-PAPR property. The development of the transceiver involves the following steps. First, the data stream is mapped into parallel QPSK-FSOK symbols and spread simultaneously by different frequency-shifted orthogonal Chu sequences. This process provides high data rate communications and retains the orthogonality property for the different parallel spread substreams. Next, the interleaved subcarriers assignment is used for both the multiuser uplink and to combat MAI interference. The Chu sequence has a constant envelope property in terms of both the frequency and time domains [12,13]. In [13], it is pointed out that the DFT of a Chu sequence is a time-scaled conjugate of the original Chu sequence. Based on a single Chu sequence, the proposed sophisticated FSOK scheme can generate a group of spreading sequences for the parallel substreams of multiple users, while maintaining low PAPR and orthogonality between substreams and users, even in the presence of multipath fading channels. Unlike the existing major PAPR-limiting techniques for multi-carrier system [14-17], e.g., the partial transmit sequences (PTS) [17] and the selective mapping (SLM) [14] methods, the proposed system does not require any side information or overhead for PAPR reduction purpose. In Section 5, computer simulation will be provided to compare the PAPR property among different schemes. Finally, the receiver structure and algorithms will be derived, including the subcarriers extraction, maximum likelihood (ML) detector, symbol despreading, and demapping. It is shown that the receiver can efficiently detect the parallel QPSK-FSOK symbols and obtain the $\mathrm{M}$-ary modulation gain and frequency diversity gain.

Moreover, we investigate the multiple input single output (MISO) scenario with high link quality performance. The SISO QPSK-FSOK transceiver is extended to combine the space-time block coding (STBC) technique [18]. Simulation results show that the proposed system can retain low-PAPR and achieve better performance than the conventional SC-FDMA and WH MC-CDMA systems over multipath channels. Furthermore, computer simulation shows that the proposed MISO QPSK-FSOK MC-CDMA system with space-frequency diversity and $\mathrm{M}$-ary modulation gain can enhance system performance and outperform the conventional STBC MISO and MIMO systems.
The rest of this article is organized as follows. In Section 2, we present the SISO system block diagram and formulate the parallel QPSK-FSOK MC-CDMA scheme. In Section 3, the SISO receiver structure with the corresponding detectors is developed. In Section 4, we propose the high-quality MISO QPSK-FSOK MC-CDMA transceiver. Simulation results for the proposed systems are provided in Section 5, while conclusions are offered in Section 6.

\section{Parallel FSOK MC-CDMA system model}

Consider an uplink multiuser MC-CDMA system with a low-PAPR property over multipath channels. The overall block diagram of the proposed FSOK MC-CDMA transceiver is depicted in Figure 1. First, assume that there are $K$ active users in an uplink MC-CDMA system. Each user is assigned $P$ parallel substreams, which are used to enhance the transmission data rate and retain the low-PAPR property. Second, to achieve multiuser uplink and combat the MAI interference, different users are assigned to different sets of interleaved subcarriers, thus maintaining perfect orthogonality between multiple users.

\subsection{Single substream transmission of each user}

As shown in Figure 1a, there are $P$ substreams of the $k$ th user being transmitted simultaneously. For the $i$ th symbol block and the $p$ th substream, the transmitted data block is denoted as $\mathbf{s}_{i, p}^{k}=\left[s_{i, p}^{k}(0) \cdots s_{i, p}^{k}(R-1) s_{i, p}^{k}(R) s_{i, p}^{k}(R+1)\right]^{T}$, which has $R+2$ bits. Hence, the overall transmitted data block over $P$ substreams is $\mathbf{s}_{i}^{k}=\left[\mathbf{s}_{i, 1}^{k^{T}} \mathbf{s}_{i, 2}^{k^{T}} \cdots \mathbf{s}_{i, p}^{k^{T}} \cdots \mathbf{s}_{i, p}^{k^{T}}\right]^{T}$ with a total of $P(R+2)$ bits. The first $R$ bits $\left[s_{i, p}^{k}(0) s_{i, p}^{k}(1) \cdots s_{i, p}^{k}(R-1)\right]$ of the $p$ th substream are mapped on to one of the $N$ codes, where $N=2^{R}$. Moreover, as shown in Figure 2, the FSOK code set forms an $N \times N$ orthogonal matrix $\mathrm{C}=\left[\mathbf{c}_{0} \ldots \mathbf{c}_{m} \ldots \mathbf{c}_{N-1}\right]$, where the $m$ th code vector is expressed as

$$
\mathbf{c}_{m}=\mathbf{f}_{m} \odot \mathbf{c}_{0}
$$

with $\mathbf{c}_{0}$ being the Chu sequence [19], $\mathbf{f}_{m}$ being an $N$ point frequency-shift sequence, and $\Theta$ being the element-by-element multiplication. Thus, the $n$th element of $\mathbf{c}_{m}$ is $\mathbf{c}_{m, n}=f_{m, n} \mathrm{c}_{0, n}$ where $f_{m, n}=\exp \{-j 2 \pi(n-1) m / N\}$ and $c_{0, n}=\exp \left\{j \pi(n-1)^{2} q / N\right\}$, with $q$ and $N$ being relatively prime. The FSOK Chu sequences retain the mutual orthogonality property $\mathbf{c}_{m}^{H} \mathbf{c}_{n}=N \delta_{m-n}$, and preserve the low-PAPR property in both the frequency and time domains. It is noted that the same orthogonal code matrix $\mathbf{C}$ is used for all $K$ users to map their transmitted data. The MAI problem will be addressed later in Section 2.3. 


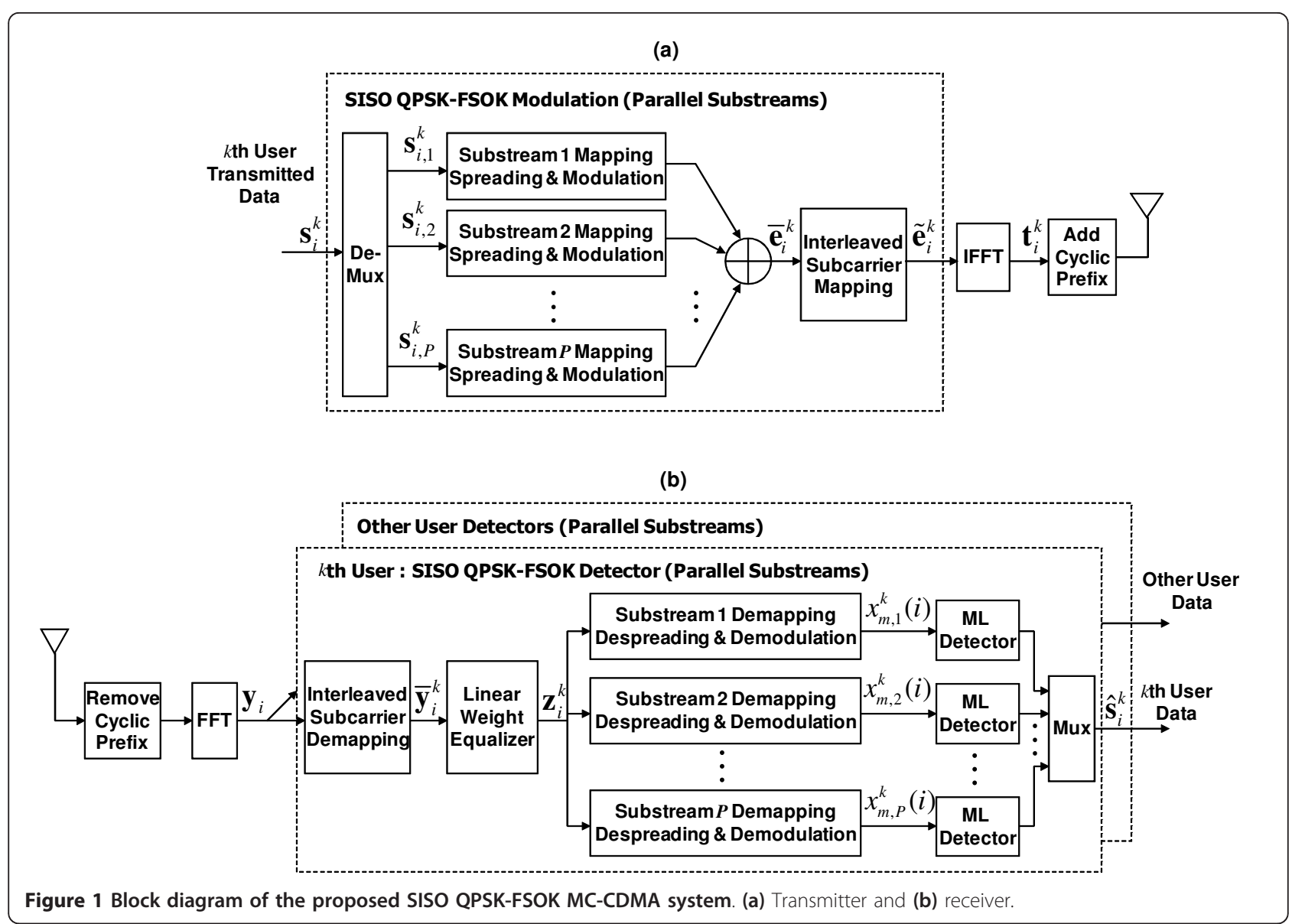

Next, as shown in Figure 2, the other two bits $\left[s_{i, p}^{k}(R) s_{i, p}^{k}(R+1)\right]$ of $\mathbf{s}_{i, p}^{k}$ are mapped on to the QPSK symbol $d_{i, p}^{k}=s_{i, p}^{k}(R)+j s_{i, p}^{k}(R+1)$, which is then spread by the $k$ th user's FSOK sequence. It is noteworthy that, to further enhance the spectrum efficiency without affecting the low-PAPR property, we can adopt M-ary phase shift keying (MPSK) for the $d_{i, p}^{k}$ symbol, with $M>$ 4. In such a case, a transmitted data block can carry a total of $P\left(R+\log _{2}(M)\right)$ bits, which will increase the spectral efficiency. Thus, the $p$ th spread QPSK-FSOK block symbol for the $k$ th user is expressed by

$$
\overline{\mathbf{c}}_{m_{i}, p}^{k}=d_{i, p}^{k} \mathbf{c}_{m_{i}, p}^{k}
$$

where $\mathbf{c}_{m_{i}, p}^{k}$ is the $m_{i}$ th mapped FSOK Chu sequence in (1), with $m_{i} \in\{0,1, \ldots, N-1\}$ being the index mapped from the first $R$ bits of the $i$ th symbol.

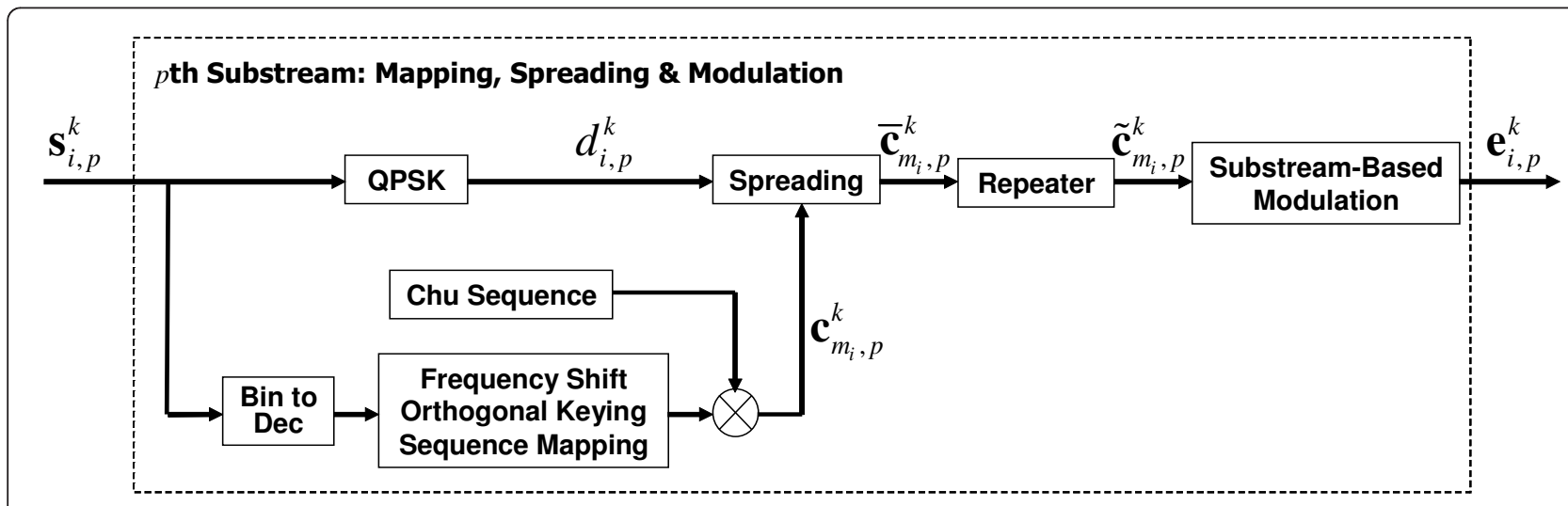

Figure 2 Block diagram of QPSK-FSOK symbol mapping, spreading, and modulation for the $p$ th substream of the kth user. 


\subsection{Parallel substream transmission of each user}

To achieve a high data rate, as shown in Figures 1a and 2 , the spread QPSK-FSOK symbol is repeated $P$ times and modulated by $\overline{\mathbf{c}}_{m_{i}, p}^{k}$. This is to transmit the $k$ th user's $P$ parallel substreams with mutual orthogonality and cope with the multiple substream interference (MSI). Its operation involves the following steps. First, for the $p$ th substream in Figure 2, the repeater is designed to duplicate the QPSK-FSOK block symbol by $P$ times, i.e.

$$
\tilde{\mathbf{c}}_{m_{i}, p}^{k}=\underbrace{\left[\overline{\mathbf{c}}_{m_{i}, p}^{k^{T}} \overline{\mathbf{c}}_{m_{i}, p}^{k^{T}} \cdots \overline{\mathbf{c}}_{m_{i}, p}^{k^{T}}\right]^{T}}_{P}=d_{i, p}^{k}\left[\mathbf{c}_{m_{i}, p}^{k^{T}} \mathbf{c}_{m_{i}, p}^{k^{T}} \cdots \overline{\mathbf{c}}_{m_{i}, p}^{k^{T}}\right]^{T}
$$

where $\tilde{\mathbf{c}}_{m_{i}, p}^{k}$ is an $N P \times 1$ vector. Next, $\tilde{\mathbf{c}}_{m_{i}, p}^{k}$ is multiplied by the $N P$-point sinusoidal modulation sequence with normalized frequency $f_{p}=P / N P$. Therefore, the repeated and modulated QPSK-FSOK symbol can be expressed by

$$
\mathbf{e}_{i, p}^{k}=\tilde{\mathbf{c}}_{m i, p}^{k} \odot \mathbf{g}_{p}=d_{i, p}^{k}{\stackrel{\mathbf{c}_{m i, p}}{k}}^{k}
$$

where

$$
\mathbf{g}_{p}=\left[1 e^{-j 2 \pi f_{p}} \cdots e^{-j 2 \pi f_{p}(N P-1)}\right]^{T}
$$

$\widehat{\mathbf{c}}_{m i, p}^{k}=\left[\widehat{\mathbf{c}}_{m i, p}^{k^{T}} \mathbf{c}_{m_{i}, p}^{k^{T}} \cdots \mathbf{c}_{m_{i}, p}^{k^{T}}\right]^{T} \odot \mathbf{g}_{p}$. It is noted that the

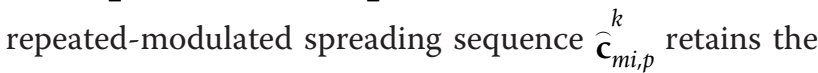
mutual orthogonality property among different substreams (see Appendix A for details), i.e.,

$$
\widehat{\mathbf{c}}_{m_{i}, p}^{k^{H}} \widehat{\mathbf{c}}_{m_{i, p}}^{k}=\left\{\begin{array}{c}
N P, \text { for } p=q \\
0, \text { for } p \neq q .
\end{array}\right.
$$

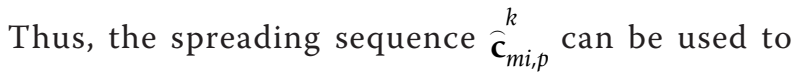
overcome the MSI and enhance the transmission data rate of the $k$ th user, that is, combining the $P$ substreams shown in Figure 1a, the composite NP-point frequencydomain signal of the $k$ th user can be expressed by

$$
\overline{\mathbf{e}}_{i}^{k}=\sum_{p=1}^{P} \mathbf{e}_{i, p}^{k}
$$

\subsection{Subcarrier assignment for multiuser uplink transmission}

As shown in Figure 1a, to provide a multiuser uplink, the composite transmission signal $\overline{\mathbf{e}}_{i}^{k}$ for $k=1,2, \ldots, K$ is assigned to different sets of interleaved subcarriers, similar to IFDMA [3]. This can maintain the low-PAPR transmission property for the $k$ th user. For $\overline{\mathbf{e}}_{i}^{k}$, the resultant interleaved NPK-point signal becomes

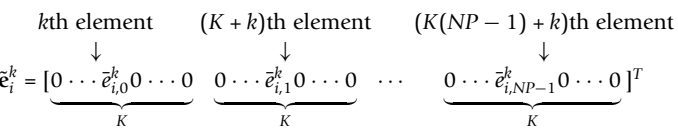

where $\tilde{\mathbf{e}}_{i}^{k}$ is an $N P K \times 1$ zero inserted vector formed by $\overline{\mathbf{e}}_{i}^{k}$ with a $(k-1)$ chip initial offset. It is noted that it involves the mutual orthogonality property for different users, i.e.,

$$
\tilde{\mathbf{e}}_{i}^{k^{H}} \tilde{\mathbf{e}}_{i}^{j}=0, \quad \text { for } k \neq j
$$

Finally, taking the IFFT of $\tilde{\mathbf{e}}_{i}^{k}$, we can form the timedomain NPK-point transmitted signal block for the $i$ th FSOK MC-CDMA symbol of the $k$ th user, i.e.,

$$
\mathbf{t}_{i}^{k}=\mathbf{Q}^{H} \tilde{\mathbf{e}}_{i}^{k}
$$

where $\mathbf{Q}^{H}$ denotes the $N P K \times N P K$ IFFT matrix. It is noted that, because of the constant modulus feature of the composite MC-CDMA FSOK Chu sequence in both the time and frequency domains, the proposed SISO MC-CDMA uplink system has a low-PAPR property. Finally, to prevent any interblock interference, a CP is inserted into each transmitted data block $\mathbf{t}_{i}^{k}$, with the length of the CP set larger than the length of the multipath channel response.

\section{Proposed parallel FSOK MC-CDMA receiver}

3.1. Channel and received signal model

After the transmitted signal is passed through the multipath channel, the circular convolution between signal and channel is induced by the use of a CP. Thus, after removing the $\mathrm{CP}$ for the multiuser scenario, the $i$ th received time-domain data block at base station can be expressed by

$$
\mathbf{r}_{i}=\sum_{k=1}^{K} \mathbf{H}^{k} \mathbf{t}_{i}^{k}+\mathbf{n}_{i}
$$

where $\mathbf{H}^{k}$ is the channel impulse response (CIR) matrix of the $k$ th user and $\mathbf{n}_{i}$ is the additive white Gaussian noise (AWGN) vector with zero-mean and variance $\sigma_{n}^{2}$. The $N P K \times N P K$ channel matrix $\mathbf{H}^{k}$ is a circulant matrix formed by cyclically shifting the zero-padded length-NPK vector of the $k$ th user CIR $\mathbf{h}^{k}=\left[h_{0}^{k} h_{1}^{k} \ldots h_{L-1}^{k}\right]^{T}$, where $L$ is the channel delay spread length. Under slow fading, we assume that $\mathbf{h}^{k}$ is invariant within a packet, but may vary from packet-topacket. Since $\mathbf{H}^{k}$ is a circulant matrix, it has the eigendecomposition $\mathbf{H}^{k}=\mathbf{Q}^{H} \boldsymbol{\Lambda}^{k} \mathbf{Q}$, where $\mathbf{Q}$ is the orthogonal FFT matrix. Further, $\Lambda^{k}$ is the diagonal element given by the NPK-point FFT of $\mathbf{h}^{k}$, i.e., $\mathbf{\Lambda}^{k}=\operatorname{diag}\left(\mathbf{Q} \mathbf{h}^{k}\right)$ with diag $\{\cdot\}$ being the diagonal matrix. 


\subsection{Development of parallel FSOK MC-CDMA receiver}

The FSOK MC-CDMA receiver is developed based on the overall block diagram depicted in Figure 1b. The receiver is designed to detect the $P$ parallel data substreams for the $K$ decoupled users simultaneously. Its operation involves the following steps. First, taking the FFT of $\mathbf{r}_{i}$, the post-FFT received signal block is given by

$$
\mathbf{y}_{i}=\mathbf{Q} \mathbf{r}_{i}=\sum_{k=1}^{K} \boldsymbol{\Lambda}^{k} \tilde{\mathbf{e}}_{i}^{k}+\overline{\mathbf{n}}_{i}=\sum_{k=1}^{K} \operatorname{diag}\left\{\mathbf{Q h} \mathbf{h}^{k}\right\} \tilde{\mathbf{e}}_{i}^{k}+\overline{\mathbf{n}}_{i}
$$

where $\overline{\mathbf{n}}_{i}=\mathbf{Q} \mathbf{n}_{i}$. Next, because of the interleaved subcarrier assignment, the post-FFT received signal $\mathbf{y}_{i}$ with $N P K \times 1$ vector can be divided into $K$ length-NP vectors. For the $k$ th user, the received vector is

$$
\overline{\mathbf{y}}_{i}^{k}=\left[y_{i, k} y_{i, k+K} \cdots y_{i, k+(N P-1) K}\right]^{T}=\overline{\boldsymbol{\Lambda}}^{k} \overline{\mathbf{e}}_{i}^{k}+\overline{\mathbf{n}}_{i}^{k}
$$

where $\overline{\boldsymbol{\Lambda}}^{k}=\operatorname{diag}\left\{\Lambda^{k}(k, k), \Lambda^{k}(k+K, k+K), \cdots, \Lambda^{k}(k+(N P-1) K, k+(N P-1) K)\right\}$, such that $\Lambda^{k}(k, k)$ is the $(k, k)$ th element of $\Lambda^{k}$ and $y_{i, k}$ is the $k$ th element of $\mathbf{y}_{i}$. Assuming that the channel response vector $\mathbf{h}^{k}$ in (11) is perfectly estimated, a linear receiver for the $k$ th user simply combines $\overline{\mathbf{y}}_{i}^{k}$ to obtain

$$
\mathbf{z}_{i}^{k}=\operatorname{diag}\left(\mathbf{w}^{k}\right)^{H} \overline{\mathbf{y}}_{i}^{k}
$$

where $\mathbf{w}^{k}$ is the combiner weight vector. For the zeroforcing weight vector, $\mathbf{w}^{k}=\mathbf{w}_{Z F}^{k}=\bar{\Lambda}^{k^{-1}}$, while in the high SNR scenario, $\mathbf{z}_{i}^{k} \approx \overline{\mathbf{e}}_{i}^{k}$. Thus, the normalized weight vector $\mathbf{w}_{Z F}^{k}$ acts as the one-tap equalizer of the proposed MC-CDMA system without requiring a matrix inversion. Moreover, to combat the noise enhancement problem, we can apply the minimum mean square error (MMSE) weight vector for the linear equalizer, i.e.,

$$
\mathbf{w}^{k}=\mathbf{w}_{\text {MMSE }}^{k}=\left[\left(\bar{\Lambda}^{k}(1,1)+\frac{1}{S N R}\right)^{-1}\left(\bar{\Lambda}^{k}(2,2)+\frac{1}{S N R}\right)^{-1} \cdots\left(\bar{\Lambda}^{k}(N P, N P)+\frac{1}{S N R}\right)^{-1}\right]^{T}
$$

where SNR is the received signal-to-noise ratio. Following linear equalization, the equalized block data of the $k$ th user can be despread by the $m$ th repeatedmodulated spreading sequence $\tilde{\mathbf{c}}_{m, p}^{k}$ of the $p$ th substream of the $k$ th user, yielding the despread output as follows

$$
\begin{aligned}
x_{m, p}^{k}(i)= & \tilde{\mathbf{c}}_{m, p}^{H} \mathbf{z}_{i}^{k} \\
= & \tilde{\mathbf{c}}_{m, p}^{H}\left\{\operatorname{diag}\left(\mathbf{w}^{k}\right)^{H} \overline{\mathbf{\Lambda}}^{k}\right\} \overline{\mathbf{e}}_{i}^{k}+\tilde{n}_{i, m, p}^{k} \\
= & \tilde{\mathbf{c}}_{m, p}^{H}\left\{\operatorname{diag}\left(\mathbf{w}^{k}\right)^{H} \overline{\mathbf{\Lambda}}^{k}\right\} \sum_{q=1}^{P} d_{i, q}^{k} \widehat{\mathbf{c}}_{m_{i, p}}^{k}+\tilde{n}_{i, m, p}^{k} \\
= & d_{i, p}^{k} \tilde{\mathbf{c}}_{m, p}^{k^{H}}\left\{\operatorname{diag}\left(\mathbf{w}^{k}\right)^{H} \overline{\boldsymbol{\Lambda}}^{k}\right\} \widehat{\mathbf{c}}_{m, p}^{k} \\
& +\tilde{\mathbf{c}}_{m, p}^{k^{H}}\left\{\operatorname{diag}\left(\mathbf{w}^{k}\right)^{H} \overline{\boldsymbol{\Lambda}}^{k}\right\} \sum_{\substack{q=1 \\
q \neq p}}^{P} d_{i, q}^{k} \widehat{\mathbf{c}}_{m_{i, p}}^{k}+\tilde{n}_{i, m, p}^{k}
\end{aligned}
$$

for $m=0,1, \ldots, N-1$, where $\tilde{n}_{i, m, p}^{k}$ is the despread noise. In (15), for the high SNR scenario with 1/SNR approaching zero, the composite equalizer-channel matrix $\left\{\operatorname{diag}\left(\mathbf{w}^{k}\right)^{H} \overline{\boldsymbol{\Lambda}}^{k}\right\}$ approximates the identity matrix. Therefore, in (15), the MSI can effectively be eliminated because of the orthogonality property in (5). Then, the despread output in (15) can be rewritten as

$$
x_{m, p}^{k}(i) \stackrel{\text { For }}{\text { High SNR }} \underset{\approx}{\approx} d_{i, p}^{k} \widehat{\mathbf{c}}_{m, p}^{k^{H}} \widehat{\mathbf{c}}_{m i, p}^{k}+\tilde{n}_{i, m, p}^{k}
$$

Moreover, the direct computation of the $N$ correlation outputs in (15) requires $\mathrm{O}\left(N^{2}\right)$ of complex multiplications. To alleviate this, an FFT/IFFT-based despreader is proposed, that is, employing the cyclic shift despreading property and some manipulation, we can express the correlation outputs as

$$
\begin{aligned}
\mathbf{x}_{p}^{k}(i) & =\left[x_{0, p}^{k}(i) \cdots x_{m, p}^{k}(i) \cdots x_{N-1, p}^{k}(i)\right]^{T} \\
& =\widehat{\mathbf{Q}}^{H} \widehat{\mathbf{C}}_{p}^{k^{H}} \widehat{\mathbf{Q}} \mathbf{z}_{i}^{k}
\end{aligned}
$$

where $\hat{\mathbf{Q}}$ is the $N P \times N P$ FFT matrix and $\widehat{\mathbf{C}}_{p}^{k^{H}}=\operatorname{diag}\left(\widehat{\mathbf{Q}}^{k}{ }^{k}\right)^{H}$ can be pre-calculated from the FFT of the base repeated-modulated spreading sequence ${ }_{\mathbf{c}_{0, p}}^{k}$. Obviously, (17) indicates that by pairwisely multiplying the two FFTs of ${ }_{\mathbf{c}_{0, p}}^{k}$ and $\mathbf{z}_{i}^{k}$ and then taking the IFFT, we obtain the desired $N$ correlator outputs $x_{m, p}^{k}(i)$ for $m$ $=0,1, \ldots, N-1$. Moreover, when $N$ is large, the computational complexity using (17) will be much lower than that associated with the original $N$-correlator bank in (15). Hence, the complexity (in number of complex multiplications) of the proposed despreader in (17) is reduced to $\mathrm{O}\left(N \log _{2} N\right)$.

Next, the $i$ th QPSK-FSOK symbol with $(R+2)$ bits of the $p$ th substream of the $k$ th user can be detected by the ML algorithm in [11]. Therefore, for the first $R$ bits, the maximizing index of the despread data $x_{m, p}^{k}(i)$ can be found by

$$
\hat{m}_{i}^{k}=\arg \max _{m}\left\{\left|\operatorname{Re}\left[x_{m, p}^{k}(i)\right]\right|+\left|\operatorname{Im}\left[x_{m, p}^{k}(i)\right]\right|\right\}, \quad 0 \leq m \leq N-1 .
$$

Based on (18), we can detect the first $R$ bits, i.e., $\left[\hat{s}_{i, p}^{k}(0) \hat{s}_{i, p}^{k}(1) \cdots \hat{s}_{i, p}^{k}(R-1)\right]^{T}=\operatorname{dec} 2 \operatorname{bin}\left\{\hat{m}_{i}^{k}\right\}$, where the function dec2bin denotes the conversion of unsigned decimal numbers into binary digits. It is noted that for the high SNR scenario, if $\hat{m}_{i}^{k}$ is a correct decision, i.e., $\hat{m}_{i}^{k}$ is equal to $m_{t}$ in (2), the maximizing value of the despreader in (15) can be approximated as 


$$
x_{\hat{m}_{i, p}^{k},}^{k} \stackrel{\substack{\text { For } \\ \text { High SNR }}}{\approx} N p d_{i, p}^{k}+n_{i, \hat{m}_{i, p}^{k}}^{k}
$$

Finally, the QPSK slicer is used for the maximal value of $\operatorname{Re}\left\{x_{\hat{m}_{i}^{k}, p}^{k}(i)\right\}$ and $\operatorname{Im}\left\{x_{\hat{m}_{i}^{k}, p}^{k}(i)\right\}$ to detect the other two bits $\left[\hat{s}_{i, p}^{k}(R) \hat{s}_{i, p}^{k}(R+1)\right]$ of the $k$ th user, respectively. From (19), it is clear that a full frequency diversity gain is obtained for the $p$ th substream of the $k$ th user. As shown in Figure 1b, the data detection scheme can be extended to all the parallel substreams and all the simultaneous users with full diversity gain by employing the different repeated-modulated spreading sequence ${\widehat{\mathbf{c}_{m, q}}}^{k}$ and different subcarrier extractions, respectively.

Through the above derivation, we have shown that the proposed transceiver can efficiently be realized and achieve MAI/MSI-free multiuser uplink transmission over multipath fading channel. Next, we verify its superior performance in terms of the matched filter bound (MFB). Assuming perfect MAI/MSI elimination, then each despread signal only contains its desired substream of the desired user and AWGN. Therefore, the matched filter's weight vector of the $k$ th user is simply given by the composite signature of the frequency-domain channel response and spreading code sequence, i.e.,

$$
\mathbf{g}_{m, p}^{k}=\Lambda^{k} \mathbf{C}_{m, p}^{k}
$$

Based on Equation 20, the maximized output SNR for the $k$ th user can be obtained as

$$
\mathrm{SNR}_{o}^{k}=\frac{\sigma_{s}^{2}}{\sigma_{n}^{2}}\left(\mathbf{c}_{m, p}^{k^{H}} \boldsymbol{\Lambda}^{k^{H}} \boldsymbol{\Lambda}^{k} \mathbf{c}_{m, p}^{k}\right)
$$

where $\sigma_{s}^{2}, \sigma_{n}^{2}$ are the desired signal and noise power, respectively, and $\mathbf{c}_{m, p}^{k^{H}} \boldsymbol{\Lambda}^{k^{H}} \boldsymbol{\Lambda}^{k} \mathbf{c}_{m, p}^{k}$ represents the processing gain because of frequency diversity combining and despreading. From the MFB in (21), an error performance bound of the $k$ th user can be evaluated and used for the verification of the superior performance of the proposed QPSK-FSOK transceiver.

\section{MISO FSOK MC-CDMA transceiver for high link quality}

In Sections 2 and 3, the SISO QPSK-FSOK MC-CDMA uplink system is proposed to achieve high data rate performance, obtain full frequency diversity gain, and preserve the low-PAPR property. In this section, an MISO extension of the QPSK-FSOK MC-CDMA uplink system is proposed to obtain the spatial diversity gain, which combines the aforementioned SISO QPSK-FSOK uplink system with an MISO STBC coding scheme. The block diagram of the proposed MISO QPSK-FSOK uplink transceiver is shown in Figure 3. Although we only discuss the simplest uplink scenario-two transmit antennas and one receive antenna, it can be easily extended to more general MIMO systems with multiple receive antennas, which can increase the spatial diversity gain.

\subsection{MISO STBC transmitter}

As shown in the MISO transmitter block diagram in Figure 3a, the $i$ th and $(i+1)$ th SISO QPSK-FSOK block symbols $\tilde{\mathbf{e}}_{i}^{k}$ and $\tilde{\mathbf{e}}_{i+1}^{k}$ of the $k$ th user can be expressed as in (7) and used for the STBC coding scheme to construct the two consecutive MISO QPSK-FSOK symbol blocks as

$$
\begin{aligned}
\overline{\mathbf{t}}_{i}^{k, 1} & =\mathbf{Q}^{H} \tilde{\mathbf{e}}_{i}^{k}, \quad \overline{\mathbf{t}}_{i+1}^{k, 1}=-\mathbf{Q}^{H} \tilde{\mathbf{e}}_{i+1}^{k^{*}} \\
\overline{\mathbf{t}}_{i}^{k, 2} & =\mathbf{Q}^{H} \tilde{\mathbf{e}}_{i+1}^{k}, \overline{\mathbf{t}}_{i+1}^{k, 2}=-\mathbf{Q}^{H} \tilde{\mathbf{e}}_{i}^{k^{*}},
\end{aligned}
$$

where the superscripts 1 and 2 are used to denote the 1st and 2nd transmit antennas, respectively.

\subsection{MISO FSOK MC-CDMA receiver}

Refer to Figure $3 \mathrm{~b}$ for the MISO receiver block diagram. After CP removal and FFT, the $i$ th and $(i+1)$ th received post-FFT symbol blocks can be expressed by

$$
\begin{aligned}
& \mathbf{y}_{i}=\sum_{k=1}^{K} \boldsymbol{\Lambda}_{i}^{k, 1} \tilde{\mathbf{e}}_{i}^{k}+\boldsymbol{\Lambda}_{i+1}^{k, 2} \tilde{\mathbf{e}}_{i+1}^{k}+\mathbf{n}_{i} \\
& \mathbf{y}_{i+1}=\sum_{k=1}^{K}-\boldsymbol{\Lambda}_{i+1}^{k, 1} \tilde{\mathbf{e}}_{i+1}^{k^{*}}+\boldsymbol{\Lambda}_{i}^{k, 2} \tilde{\mathbf{e}}_{i}^{k^{*}}+\mathbf{n}_{i+1}
\end{aligned}
$$

where $\boldsymbol{\Lambda}_{i}^{k, 1}$ and $\boldsymbol{\Lambda}_{i}^{k, 2}$ denote the channel matrices from the 1 st and 2 nd transmit antennas to the single receive antenna, respectively. Similar to (12), because of the interleaved subcarrier assignment, the two extracted signal blocks of the $k$ th user can be written as

$$
\begin{aligned}
& \overline{\mathbf{y}}_{i}^{k}=\left[y_{i, k} \gamma_{i, k+K} \cdots \gamma_{i, k(N P-1) K}\right]^{T}=\overline{\mathbf{\Lambda}}_{i}^{k, 1} \overline{\mathbf{e}}_{i}^{k}+\overline{\mathbf{\Lambda}}_{i+2}^{k, 2} \overline{\mathbf{e}}_{i+1}^{k}+\overline{\mathbf{n}}_{i}^{k} \\
& \overline{\mathbf{y}}_{i+1}^{k}=\left[y_{i+1, k} \gamma_{i+1, k+K} \cdots \gamma_{i+1, k+(N P-1) K}\right]^{T}=-\overline{\mathbf{\Lambda}}_{i+1}^{k, 1} \overline{\mathbf{e}}_{i+1}^{k^{k}}+\overline{\mathbf{\Lambda}}_{i}^{k, 2} \overline{\mathbf{e}}_{i+1}^{k^{*}}+\overline{\mathbf{n}}_{i+1}^{k}
\end{aligned}
$$

where

boldsymbol $\Lambda_{j}^{k, 1}=\operatorname{diag}\left\{\Lambda_{j}^{k, 1}(k, k), \Lambda_{j}^{k, 1}(k+K, k+K), \cdots, \Lambda_{j}^{k, 1}(k+(N P-1) K, k+(N P-1) K)\right\} \quad$ and $\bar{\Lambda}_{j}^{k, 2}=\operatorname{diag}\left\{\Lambda_{j}^{k, 2}(k, k), \Lambda_{j}^{k, 2}(k+K, k+K), \cdots, \Lambda_{j}^{k, 2}(k+(N P-1) K, k+(N P-1) K)\right\}$ for $j=i, i+1$. Assume that the two spatial channels are fixed over two consecutive blocks, i.e., $\overline{\boldsymbol{\Lambda}}_{i}^{k, 1}=\overline{\boldsymbol{\Lambda}}_{i+1}^{k, 1}=\overline{\boldsymbol{\Lambda}}^{k, 1}$ and $\overline{\boldsymbol{\Lambda}}_{i}^{k, 2}=\overline{\boldsymbol{\Lambda}}_{i+1}^{k, 2}=\overline{\boldsymbol{\Lambda}}^{k, 2}$ for $k=1,2, \ldots, K$. From (24), the cascaded received data can be formed as

$$
\tilde{\mathbf{y}}_{i}^{k}=\left[\begin{array}{c}
\overline{\mathbf{y}}_{i}^{k} \\
\overline{\mathbf{y}}_{i+1}^{k^{*}}
\end{array}\right]=\left[\begin{array}{cc}
\overline{\boldsymbol{\Lambda}}^{k, 1} & \overline{\boldsymbol{\Lambda}}^{k, 2} \\
\overline{\boldsymbol{\Lambda}}^{k, 2^{*}} & -\overline{\boldsymbol{\Lambda}}^{k, 1^{*}}
\end{array}\right]\left[\begin{array}{c}
\overline{\mathbf{e}}_{i}^{k} \\
\overline{\mathbf{e}}_{i+1}^{k}
\end{array}\right]+\left[\begin{array}{c}
\overline{\mathbf{n}}_{i}^{k} \\
\overline{\mathbf{n}}_{i+1}^{k^{*}}
\end{array}\right]
$$

Noting the orthogonal structure of the composite channel matrix in (25), a simple maximum ratio combiner (MRC) can be used to obtain the spatial diversity 


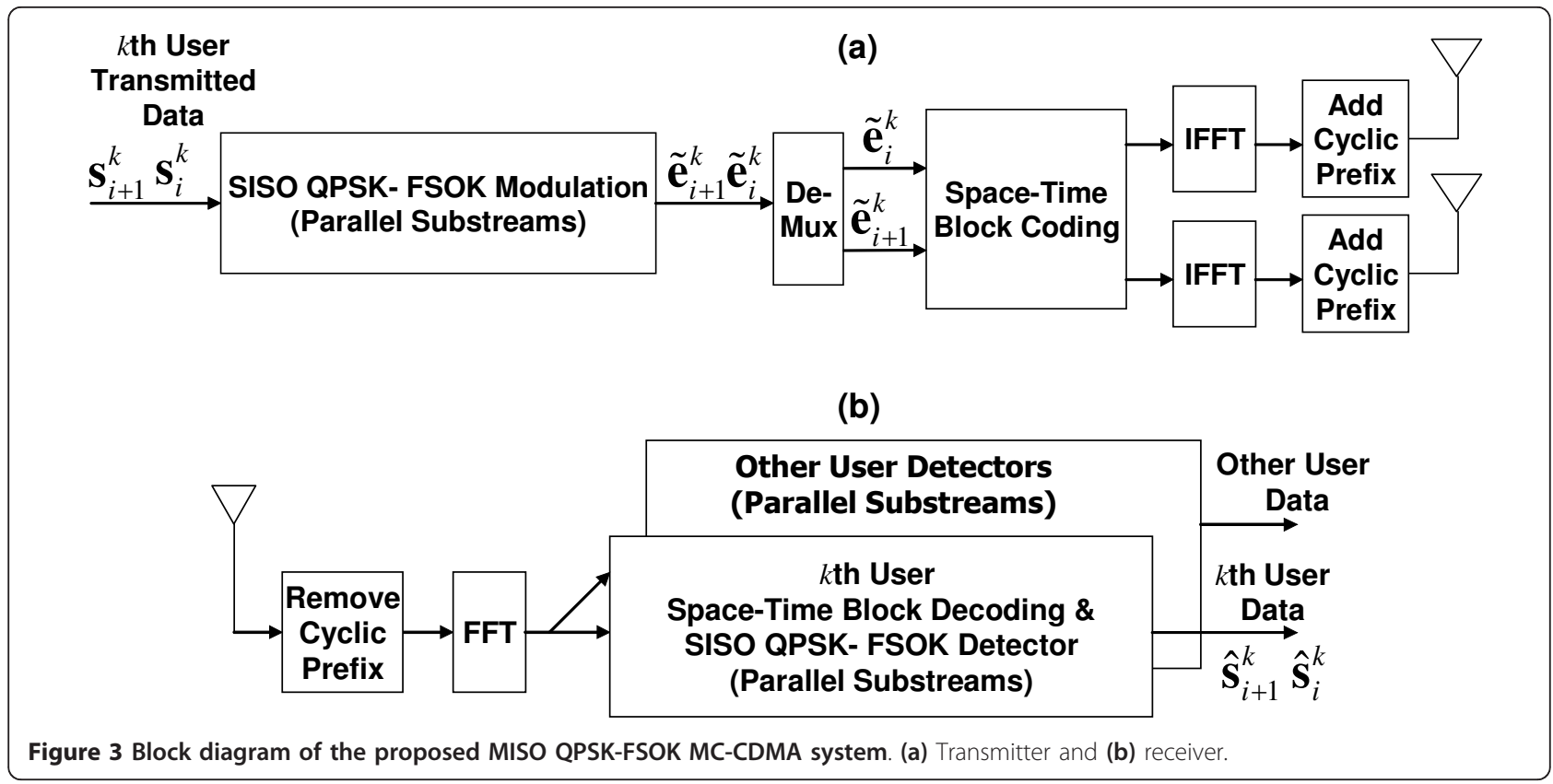

gain, i.e.,

$$
\begin{aligned}
& \mathbf{u}_{i}^{k}=\mathbf{V}_{1}^{k^{H}} \tilde{\mathbf{y}}_{i}^{k}=\tilde{\Lambda}^{k} \overline{\mathbf{e}}_{i}^{k}+\tilde{\mathbf{n}}_{i}^{k} \\
& \mathbf{u}_{i+1}^{k}=\mathbf{V}_{1}^{k^{H}} \tilde{\mathbf{y}}_{i}^{k}=\tilde{\Lambda}^{k} \overline{\mathbf{e}}_{i+1}^{k}+\tilde{\mathbf{n}}_{i+1}^{k}
\end{aligned}
$$

where the MRC weight vectors are given by $\mathbf{V}_{1}^{k}=\left[\overline{\boldsymbol{\Lambda}}^{k, 1^{T}} \overline{\boldsymbol{\Lambda}}^{k, 2^{H}}\right]^{T}$ and $\mathbf{V}_{2}^{k}=\left[\overline{\boldsymbol{\Lambda}}^{k, 2^{T}}-\overline{\boldsymbol{\Lambda}}^{k, 1^{H}}\right]^{T} ; \tilde{\mathbf{n}}_{i}^{k}$ and $\tilde{\mathbf{n}}_{i+1}^{k}$ are the post-MRC noise vectors; and $\tilde{\boldsymbol{\Lambda}}^{k}=\overline{\boldsymbol{\Lambda}}^{k, 1^{H}} \overline{\boldsymbol{\Lambda}}^{k, 1}+\overline{\boldsymbol{\Lambda}}^{k, 2^{H}} \overline{\boldsymbol{\Lambda}}^{k, 2}$ is a diagonal matrix with the $(n, n)$ th element being $\left|\bar{\Lambda}^{k, 1}(n, n)\right|^{2}+\left|\bar{\Lambda}^{k, 2}(n, n)\right|^{2}$.

Similarly, from (13) in the SISO system, the linear receiver $\mathbf{w}^{k}$ for the $k$ th user can be used to equalize the channel effect, yielding the two consecutive data blocks, i.e., $\overline{\mathbf{z}}_{i}^{k}=\operatorname{diag}\left(\mathbf{w}^{k}\right)^{H} \mathbf{u}_{i}^{k}$ and $\overline{\mathbf{z}}_{i+1}^{k}=\operatorname{diag}\left(\mathbf{w}^{k}\right)^{H} \mathbf{u}_{i+1}^{k}$ with $\mathbf{w}^{k}$ being ZF or MMSE vectors shown in (14). Next, to suppress the MSI, the $i$ th and $(i+1)$ th equalized data blocks can be despread by the two independent repeated-modulated spreading sequences $\widehat{\mathbf{c}}_{m_{1}, p}^{k}$ and $\widehat{\mathbf{c}}_{m_{2}, p}^{k}$ to get the two outputs $x_{m_{1}, p}^{k}(i)={\widehat{\mathbf{c}_{m}, p}}_{k_{i}^{H}}^{\overline{\mathbf{z}}_{i}^{k}}$ and $x_{m_{2}, p}^{k}(i+1)=\widehat{\mathbf{c}}_{m_{2}, p}^{k^{H}} \overline{\mathbf{z}}_{i}^{k}$ for $m_{1}, m_{2}=0,1, \ldots, N$, which is similar to (15). Finally, the ML algorithm in (18) can be used to demap and detect the two consecutive MISO QPSK-FSOK symbols of the $p$ th substream, i.e., $\left[\hat{s}_{i, p}^{k}(0) \hat{s}_{i, p}^{k}(1) \cdots \hat{s}_{i, p}^{k}(R+1)\right]^{T}$ and $\left[\hat{s}_{i+1, p}^{k}(0) \hat{s}_{i+1, p}^{k}(1) \cdots \hat{s}_{i+1, p}^{k}(R+1)\right]^{T}$. For the high SNR scenario, if correct decisions regarding the two frequency shifts have been made, i.e., $\hat{m}_{1}^{k}=m_{i}$ and $\hat{m}_{2}^{k}=m_{i+1}$, then the maximizing values of the despreader for the $i$ th and $(i+1)$ th symbol blocks are given by $x_{\hat{m}_{1, p}^{k}}^{k}(i) \stackrel{\substack{\text { For } \\ \text { High SNR }}}{\approx} N P d_{i, p}^{k}$ and $x_{\hat{m}_{2, p}^{k}}^{k}(i+1) \stackrel{\substack{\text { For } \\ \text { High SNR }}}{\approx} N P d_{i+1, p}^{k}$ for $p=q$. In such a way, we can detect all substreams for all users with full spatial and frequency diversity gain. Moreover, for the downlink system, the mobile user can acquire the spatial and frequency diversity gain from the BS with a two-antenna transmission downlink.

\section{Computer simulations}

In this section, simulation results are demonstrated to confirm the performance of the proposed parallel FSOK MC-CDMA system. The environment considered is the uplink of a simplified single cell system over multipath channels. For all simulations, a quasi-static multipath fading channel is assumed during each packet, as well as independence between packets. To test the system under a severe multipath channel environment, we assume that the channel profile has $L$ independent frequency selective Rayleigh fading paths with equal power and time delays randomly chosen from $\left[0,(G-1) T_{\mathrm{s}}\right]$, where $T_{\mathrm{s}}$ is the sampling time and the CP length is $G=(N P K) / 4$. Hence, the fading gains can be generated from the independent, identically distributed (i.i.d.) complex Gaussian random variables with zero mean and unity variance [20]. For the proposed parallel QPSK-FSOK MC-CDMA system, one symbol contains $\left(\log _{2} N+2\right) P$ bits for each user. Next, as a performance index, the bit error rate (BER) is evaluated at different $E_{\mathrm{b}} / N_{0}$. Unless otherwise mentioned, the following parameters are assumed: $N=8, P=4, K=4, G=$ $32, L=4, E_{\mathrm{b}} / N_{0}=10 \mathrm{~dB}$, and NFR $=0 \mathrm{~dB}$, where the NFR (near-far-ratio) is defined as the ratio of MAI power 
to signal power. For performance comparisons, BER simulations are conducted for the proposed QPSK-FSOK MC-CDMA, conventional MC-CDMA [8], interleaved SC-FDMA [6], and ideal QPSK systems [21,22]. The BER for the ideal QPSK system is evaluated using the ideal matched filter for multipath channels. The conventional MC-CDMA and interleaved SC-FDMA systems consist of the $K P$ length- $M$ and length- $Q \mathrm{WH}$ codes, respectively, where there are for $K$ users and each user is transmitting data over $P$ subcarriers. The above three systems can provide the same data rate. For the proposed method in (17), the order of computational complexity will be $O$ $\left(N \log _{2} N\right)$ because of the despreader and ML detector. However, the conventional MC-CDMA system [8] utilizes the MMSE equalizer, which requires a matrix inversion operation, resulting in a computational order of $O$ $\left(N^{3}\right)$. For SC-FDMA, employing a well-known frequencydomain one-tap equalizer, its complexity order is $O(N)$. Although the SC-FDMA receiver has the lowest complexity, its BER performance is significantly inferior to the proposed system, as shown in the following simulation results. Therefore, the proposed system provides a good compromise in terms of complexity and performance.

In the first simulation, the BER performance is evaluated as a function of $E_{\mathrm{b}} / N_{0}$ for the proposed system over the varying multipath number $L$. In Figure 4 , it is shown that as the multipath number $L$ increases, the proposed system obtains greater diversity gain and provides higher link quality performance. Figure 4 also shows that the proposed QPSK-FSOK MMSE system with multipath diversity gain leads to better performance as compared to the conventional MC-CDMA and interleaved SC-FDMA systems under the same data rate scenario. For example, the proposed QPSK-FSOK system $(N=4, P=8, K=4)$ with $\left(\log _{2} N+2\right) P K=128$ bits/sym, the conventional MC-CDMA system $(M=$ $128, P=16, K=4)$ with $2 K P=128$ bits $/$ sym, and the interleaved SC-FDMA system $(Q=2, P=16, K=4)$ with $2 K P=128 \mathrm{bits} / \mathrm{sym}$ all have the same user data rates and total number of subcarriers $M=N P K=Q P K$ $=128$. In this simulation, it is confirmed that the proposed system can obviate MAI, MSI, and MPI at the same time.

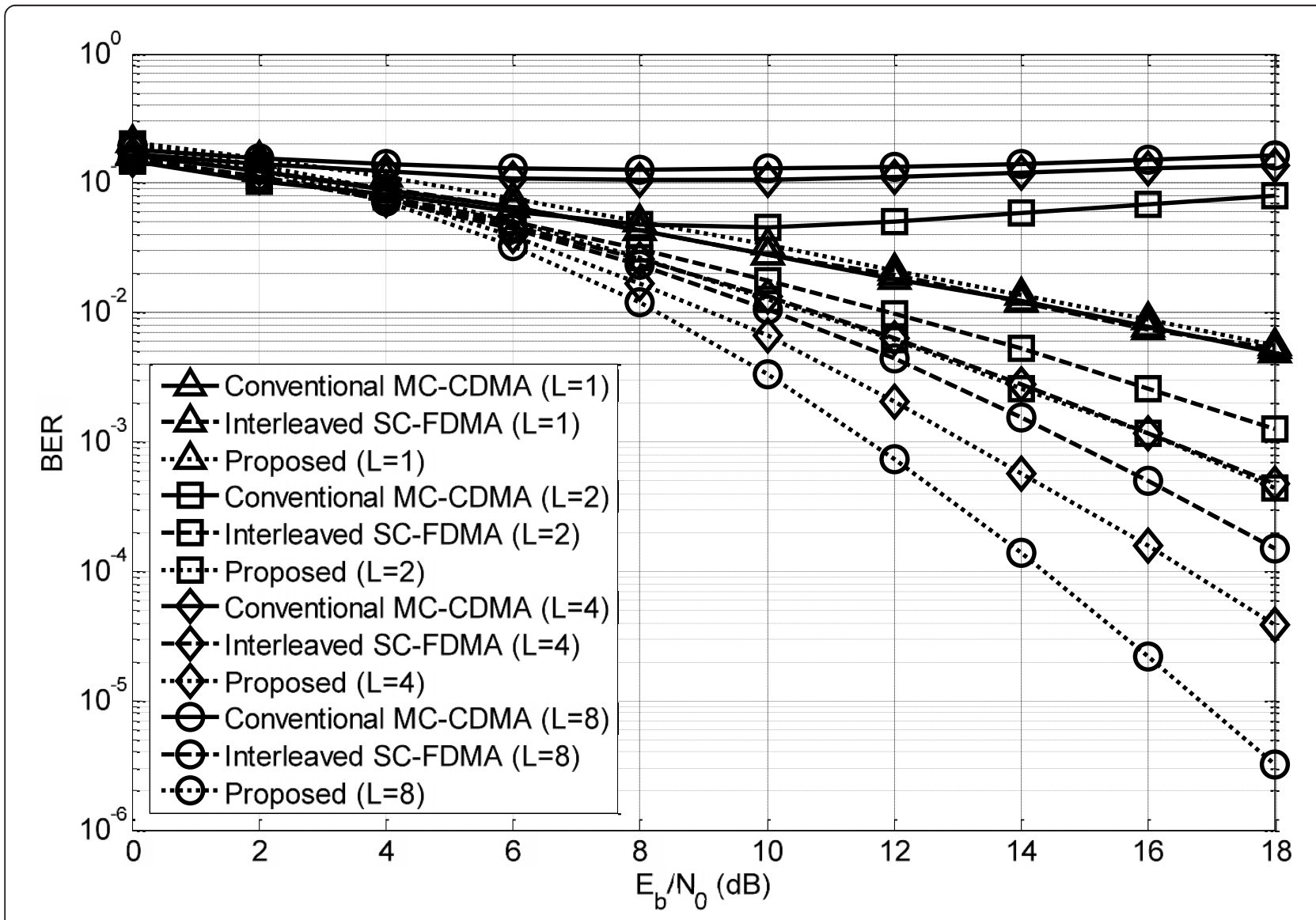

Figure 4 BER performance comparison of the proposed MMSE FSOK MC-CDMA, the interleaved SC-FDMA, and the conventional MCCDMA systems for $K=4$ users over varying number of equal-power multipath channels $(L=1,2,4,8)$. 
In the second simulation, the M-ary modulation gain and multipath diversity gain are demonstrated for the proposed system using an MMSE receiver with a varying parameter $N$, and fixed parameters $P=4, K=4$, and $E_{\mathrm{b}} /$ $N_{0}=12 \mathrm{~dB}$. To combat the MPI, the BER for different symbol lengths $(N P K)$ of the QPSK-FSOK block symbol is evaluated, e.g., $L=8$, symbol length $\geq 4 L=32$. Figure 5 shows that the BER of the proposed system successively improves as the FSOK and multipath lengths increase. Besides, to verify the error performance bound, the BER bound corresponding to the MF weight vector in (20) is evaluated and shown in Figure 5 for different multipath order $L$ and spreading code length $N$. It is seen that for the $L=4$ scenario, the proposed system can approach the MFB performance with diversity order $L=4$, that is, as $N$ increases, the proposed transceiver can efficiently combat the MSI and MAI and approach the optimal BER performance given by the MFB. Moreover, for large $N$, the proposed system can ever outperform the theoretical QPSK BER performance. Because the proposed system can acquire both the M-ary modulation gain in terms of the spreading code length $N$ and full diversity gain in terms of the multipath order $L$, as (21) indicates. On the other hand, the ideal QPSK BER performance [22] exhibits only the full frequency diversity gain because of the multipath propagation, but without the M-ary modulation gain.

In the third simulation, the BER performance of the proposed QPSK-FSOK MMSE system for different $P$ and $N$ is shown in Figure 6. We find that at different data rates under the same symbol length $(N P K)$, the low-rate configuration of the proposed system $(P=1$ and $N=32$ ) can outperform the high-rate configuration $(P=32$ and $N=1)$ by about $6 \mathrm{~dB}$ at BER $=1 \times 10^{-3}$. This confirms that as $N$ increases for the $L=4$ scenario, the $\mathrm{M}$-ary modulation gain can assist the proposed system to approach the theoretical QPSK performance.

In the fourth simulation, we consider the BER performance of the proposed high link quality MISO and MIMO QPSK-FSOK transceivers. First, we set the number of multipath channels at $L=4$ and verify that the high link quality performance for the two transmit antennas and single receive antenna (2Tx, $1 \mathrm{Rx})$ has a spatial diversity order of 2. As shown in Figure 7, the proposed MISO MC-CDMA transceiver outperforms the theoretical QPSK SIMO MRC (1Tx, 2Rx) system by about $2 \mathrm{~dB}$ at BER $=1 \times 10^{-4}$. Moreover, the proposed high link quality MISO and MIMO transceivers can outperform the conventional QPSK STBC MISO (2Tx, 1Rx) and MIMO $(2 \mathrm{Tx}, 2 \mathrm{Rx})$ systems after $E_{\mathrm{b}} / N_{0}$ becomes

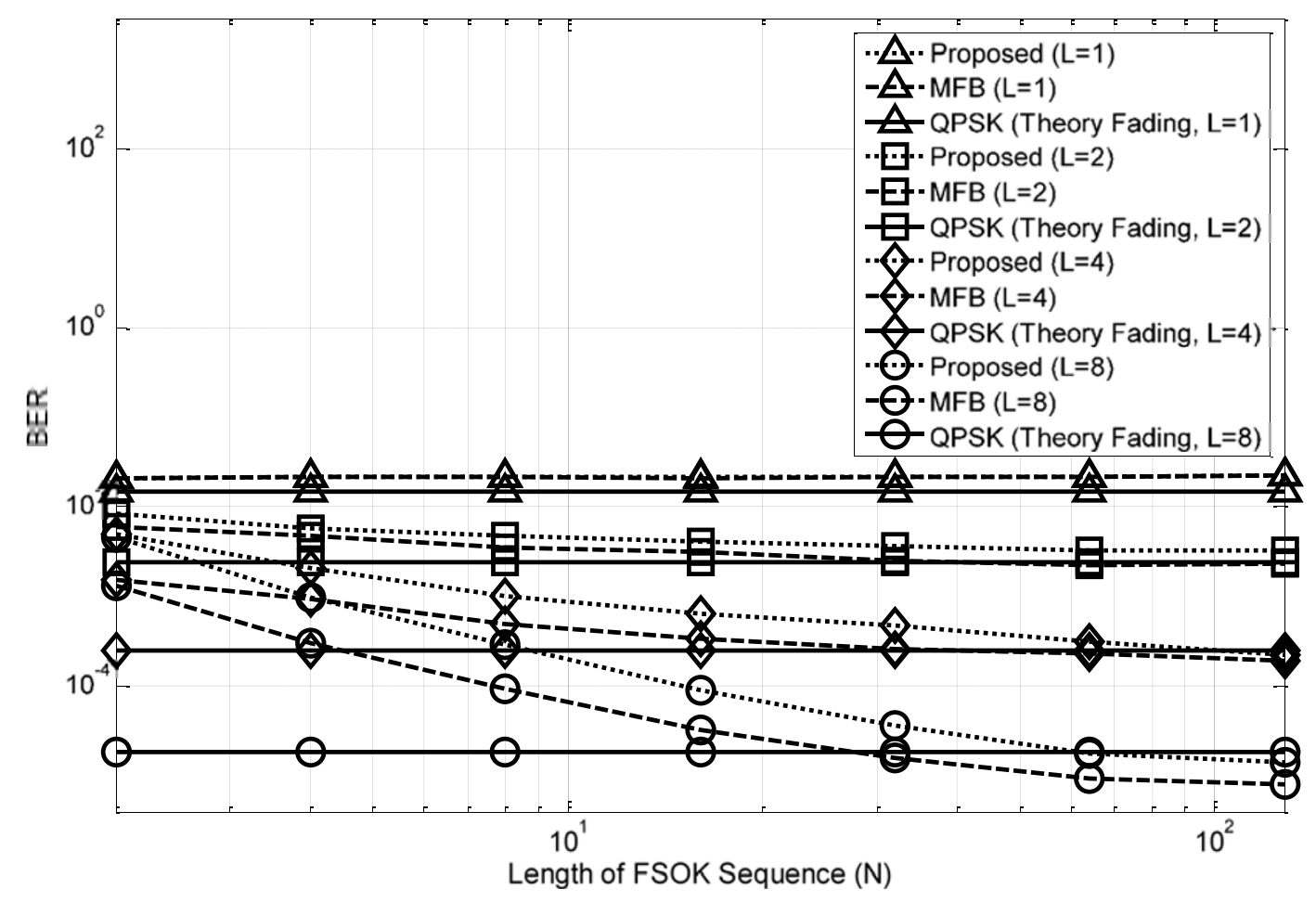

Figure 5 BER performance comparison of the theoretical QPSK and the proposed MC-CDMA systems for different FSOK sequence lengths $(N)$ and multipath numbers $(L=1,2,4,8)$ at $E_{\mathrm{b}} / N_{0}=12 \mathrm{~dB}$. 


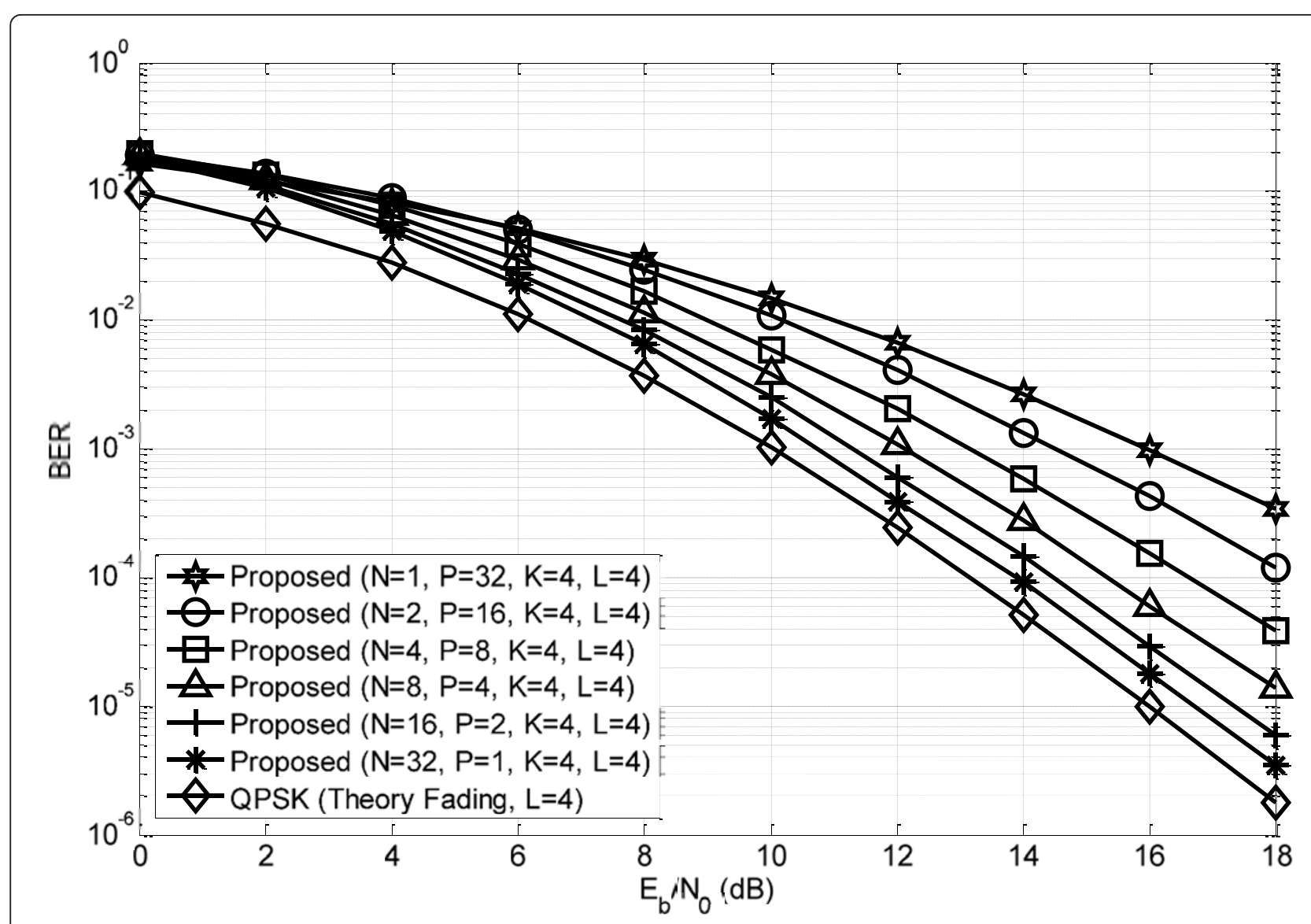

Figure 6 BER performance comparison of the theoretical QPSK and the proposed MC-CDMA systems with different data rates (different number of parallel substreams).

larger than 10 and $8 \mathrm{~dB}$, respectively. Therefore, we note that the proposed STBC MISO and MIMO systems are superior to the conventional STBC MISO and MIMO systems, because of the threefold effect of the M-ary FSOK modulation gain, spatial diversity gain, and multipath diversity gain.

Finally, we evaluate the PAPR property of the transmitted QPSK-FSOK signal with an oversampling factor of 4 for the raised-cosine pulse-shaping filter interpolation. The PAPR (in $\mathrm{dB}$ ) is defined as

$$
\operatorname{PAPR}_{\mathrm{dB}}=10 \log _{10}\left(\frac{\max \left\{\left|x_{k}\right|^{2}\right\}}{\left\langle\left|x_{k}\right|^{2}\right\rangle}\right)
$$

where $x_{k}$ is the $k$ th interpolated sample, and $\langle\cdot\rangle$ denotes the time-average operation. For the proposed MC-CDMA, the interleaved/localized SC-FDMA, the conventional MC-CDMA systems, and the conventional PTS [17] and SLM [14] schemes for multi-carrier systems, the complementary cumulative distribution function (CCDF) of the PAPR is plotted in Figure $8 \mathrm{a}, \mathrm{b}$ under two different roll-off factors of 0.5 and 0.35 , respectively. The number of sub-carriers is chosen to be 128 for all the systems. As shown in Figure $8 \mathrm{a}, \mathrm{b}$, the transmit signal of the proposed system has lower PAPR than the conventional PTS and SLM schemes. Besides, the localized SC-FDMA has a higher PAPR than the interleaved SC-FDMA, as shown in previous study [1]. We further observe that the proposed FSOK MC-CDMA system exhibits a lower PAPR than the conventional WH MC-CDMA and localized SC-FDMA systems. All three of the above systems employ different spreading schemes in terms of frequency-domain to extract the frequency diversity gain. However, regarding the aspect of PAPR reduction, the spreading schemes of the latter two systems are not as effective as that of the proposed FSOK system, which cleverly exploits the Chu sequence properties. Thus, the proposed system is less demanding in terms of power amplifier linearity.

\section{Conclusions}

In this article, we propose a new low-PAPR FSOK MCCDMA transceiver that is suitable for uplink 


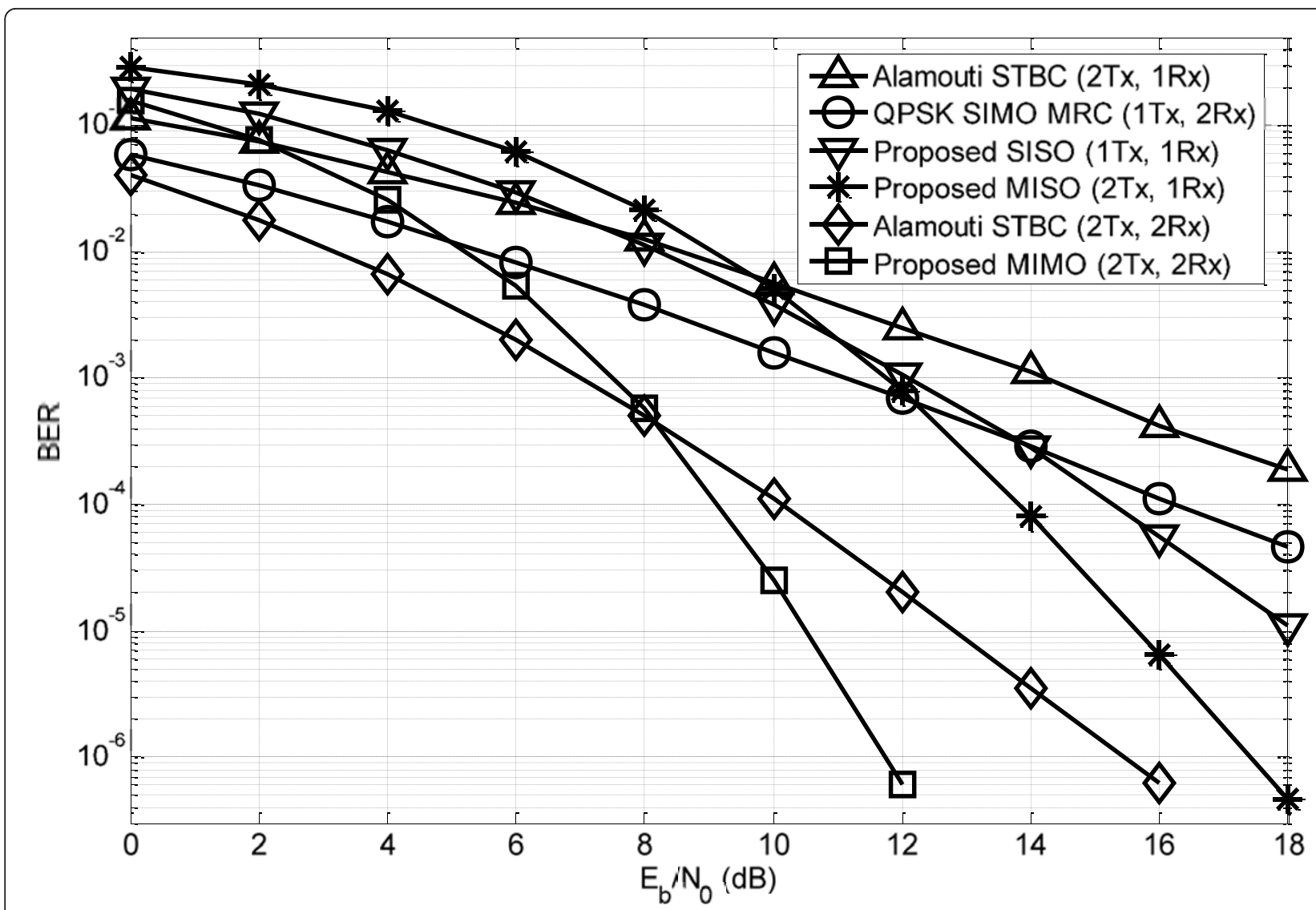

Figure 7 BER performance comparison of the proposed SISO/MISO/MIMO MC-CDMA system and Alamouti MISO/MIMO STBC system over multipath fading channels $(L=4)$.

communications with a high data rate and high performance over multipath fading channels. First, for a multiuser uplink, the parallel FSOK scheme with interleaved subcarrier assignments is designed to provide multiuser transmissions with a constant envelope property, and to combat MAI and MPI. At the receiver, an efficient ML algorithm with FSOK despreader and demapper is used to detect the modulation symbols, which can obtain the $\mathrm{M}$-ary modulation gain and diversity gain. Moreover, we propose the extended MISO FSOK uplink configuration to acquire a high link quality. Simulation results show that the proposed multiuser uplink system outperforms the conventional MC-CDMA and SC-FDMA systems.

\section{Appendix A}

Derivation of the mutual orthogonality property for the repeated-modulated spreading sequence $\hat{\mathbf{c}}_{m_{i}, p}^{k}$

From (4), we have the repeated-modulated spreading sequence as $\widehat{\mathbf{c}}_{m_{i}, p}^{k}=\left[\mathbf{c}_{m_{i}, p}^{k^{T}} \mathbf{c}_{m_{i}, p}^{k^{T}} \cdots \mathbf{c}_{m_{i}, p}^{k^{T}}\right]^{T} \odot \mathbf{g}_{p}$. Therefore, their inner product can be calculated as

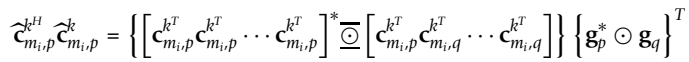

$$
\begin{aligned}
& =\left[\mathbf{f}_{m_{i}, q-m_{i},}^{\mathbf{k}^{T}} \mathbf{f}_{m_{i}, q-m_{i}, p}^{k^{T}} \cdots \mathbf{f}_{m_{i}, q-m_{i}, p}^{T^{T}}\right] \mathbf{g}_{q-p}^{T} \\
& =\sum_{l=0}^{P-1} \sum_{n=0}^{N-1} e^{-j 2 \pi \frac{m_{i}}{N}} e^{-j 2 \pi \frac{\mu}{N}(I N+n)} \\
& =\sum_{l=0}^{P-1} e^{-j 2 \pi \mu l} \sum_{n=0}^{N-1} e^{-j 2 \pi \frac{\left(\bar{m}_{i}+\mu\right)}{N} n}
\end{aligned}
$$

where $\bar{m}_{i}=\left(m_{i}, q\right)-\left(m_{i}, q\right)$ is the difference between the two CSOK symbol indices of the $q$ th and $p$ th substreams, and $\mu=(q-p) / P$. Next, for the case $p \neq q$, we have

$$
\sum_{l=0}^{P-1} e^{-j 2 \pi \mu l}=0
$$

such that the inner product in (28) is zero for $p \neq q$. For the case $p=q$, we have $\mu=\bar{m}_{i}=0$, and

$$
\sum_{l=0}^{P-1} e^{-j 2 \pi \mu l}=P, \quad \sum_{n=0}^{N-1} e^{-j 2 \pi \frac{\left(\bar{m}_{i}+\mu\right)}{N} n}=N
$$




\section{(a)}

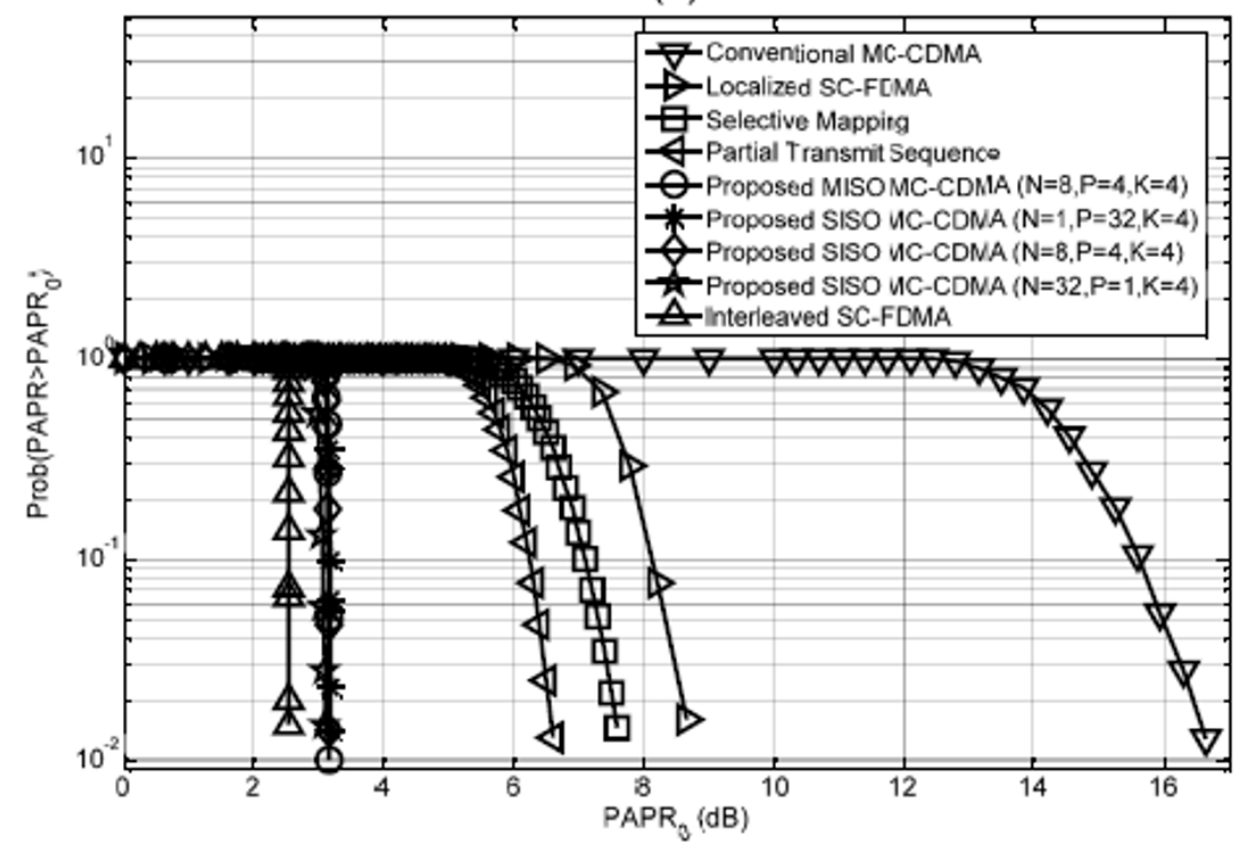

(b)

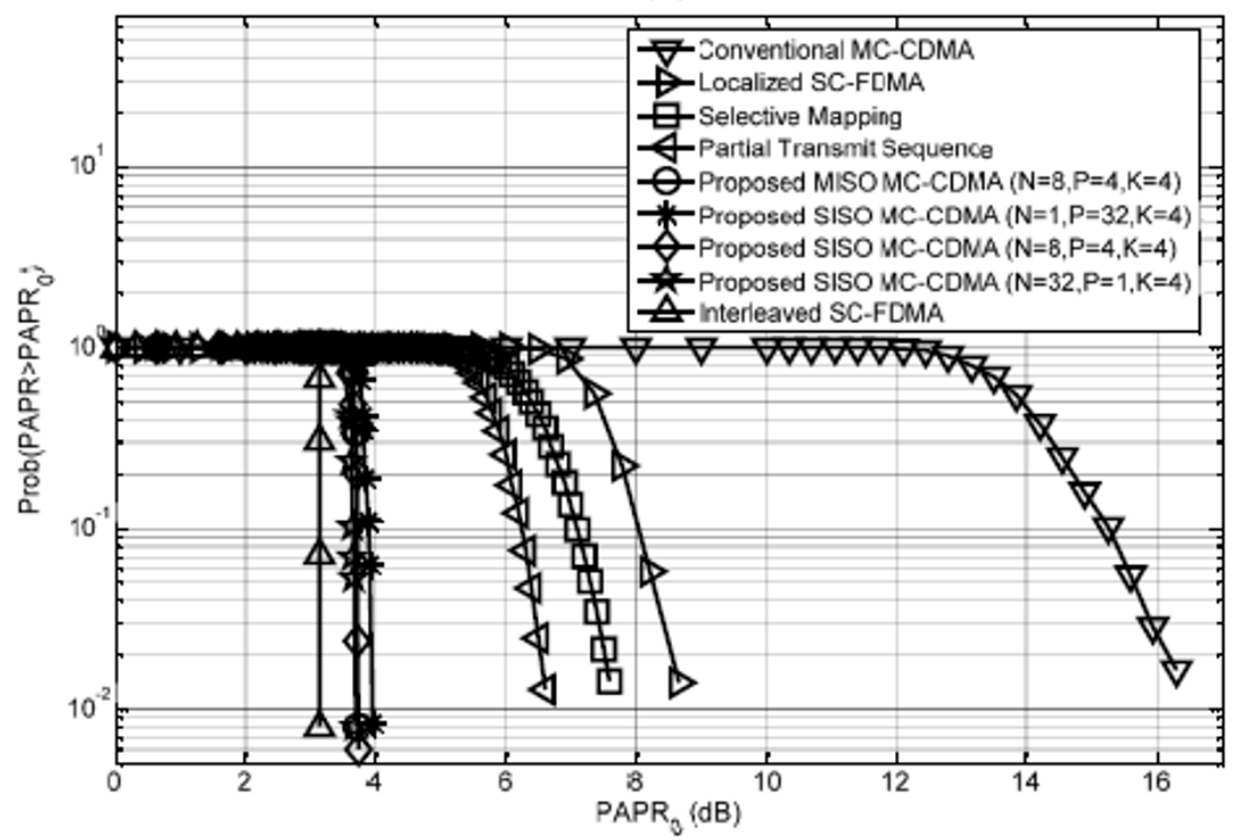

Figure 8 Comparison of the PAPR CCDF for the proposed MC-CDMA, the conventional SC-FDMA, the conventional MC-CDMA systems, the conventional SLM, and PTS schemes. (a) 50\% roll-off. (b) 35\% roll-off.

Therefore, from (28) to (30), the orthogonality in (5) can be derived

$$
\widehat{\mathbf{c}}_{m_{i}, p}^{k^{H}} \widehat{\mathbf{c}}_{m_{i}, p}^{k}= \begin{cases}N P, & \text { for } p=q \\ 0, & \text { for } p \neq q\end{cases}
$$

\section{Appendix B}

Proof of the constant envelope of transmit signal $\mathbf{t}_{i}^{k}$

The time-domain NPK-point transmitted signal block of the $i$ th FSOK MC-CDMA symbol of the $k$ th user in (9) can be expressed by 


$$
\mathbf{t}_{i}^{k}=\mathbf{Q}^{H} \tilde{e}_{i}^{k}
$$

For simplicity, we consider the first user $(k=1)$. From (32) and (7), the $\tilde{m}$ th element of the first user's timedomain sequence $\mathbf{t}_{i}^{1}$ can be derived as follows

$$
t_{i}^{1}(\tilde{m})=\sum_{l=0}^{N P K-1} \tilde{e}_{i, e^{1}}^{j 2 \pi \tilde{m} /(N P K)}=\sum_{n=0}^{N P-1} \bar{e}_{i, n}^{1} e^{j 2 \pi \tilde{m} n /(N P)}, \quad \text { for } \tilde{m}=0,1, \cdots, N P K-1
$$

where $t_{i}^{1}(\tilde{m})$ has a repetition property in that $t_{i}^{1}(\tilde{m})=t_{i}^{1}(m+N P)=\cdots=t_{i}^{1}(m+K N P)$ for $m=0,1, \ldots$, $N P$ - 1. In the following, we will verify the constant envelope property for the first repetition $\left\{t_{i}^{1}(m)=0,1, \cdots, N P-1\right\}$. Let $\tilde{e}_{i, l}^{1}$ be the $l$ th element of $\tilde{\mathbf{e}}_{i}^{1}$ in (7) with zero insertion, i.e.,

$$
\tilde{e}_{i, l}^{1}= \begin{cases}\bar{e}_{i, l / K^{\prime}}^{1} l=n K, & 0 \leq n \leq N P-1 \\ 0, & \text { otherwise }\end{cases}
$$

with $\bar{e}_{i, n}^{1}$ being the $n$th element of $\overline{\boldsymbol{e}}_{i}^{1}$ in (6). Based on (1)-(5), the $\bar{e}_{i, n}^{1}$ can be rewritten as

$$
\bar{e}_{i, n}^{1}=\bar{e}_{i, u, v}^{1}=\sum_{p=1}^{P} d_{i, p}^{1} e^{j \pi v^{2} q / N} e^{-j 2 \pi m_{i, p} v / N} e^{-j 2 \pi p(u N+v) /(N P)}
$$

where $n=u N+v, u=0,1, \ldots, P-1$, and $v=0,1, \ldots$, $N$ - 1 . Next, substituting (35) into (33), $t_{i}^{1}(m)$ can be derived by

$$
\begin{aligned}
& t_{i}^{1}(m)=\sum_{p=1}^{P} d_{i, p}^{1}\left\{\sum_{u=0}^{P-1} \sum_{v=0}^{N-1} e^{j \pi \pi v^{2} \alpha / N} e^{-j 2 \pi m i, p / N} e^{-j 2 \pi p(u N+v) /(N P)} e^{j 2 \pi m(u N+v) /(N P)}\right\}
\end{aligned}
$$

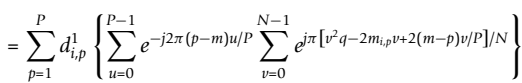

where the Chu sequence parameters $q$ and $N$ are relatively prime. For simplicity, we assume the case of $q=1$ and an even $N$. Therefore, $t_{i}^{1}(m)$ in (36) can be rewritten as

$$
t_{i}^{1}(m)=\sum_{p=1}^{P} d_{i, p}^{1} e^{-j \pi \rho^{2} / N}\left\{\sum_{u=0}^{P-1} e^{-j 2 \pi(p-m) u / P}\right\}\left\{\sum_{v=0}^{N-1} e^{j \pi[v-\rho]^{2} / N}\right\}
$$

where $\rho=m_{i, p}-(m-p) / P$. When $m=p, \rho=m_{i, p}$ is an integer and

$$
\sum_{u=0}^{P-1} e^{-j 2 \pi(p-m) u / P}=\left\{\begin{array}{l}
P, p=m \\
0, p \neq m
\end{array}\right.
$$

According to [12], we use the following identity for any integer $\rho$ :

$$
\sum_{v=0}^{N-1} e^{j \pi[v-\rho]^{2} / N}=\sum_{v=0}^{N-1} e^{j \pi v^{2} / N}=e^{j \pi / 4} \sqrt{N}
$$

Therefore, substituting (38) and (39) into (37), we can obtain

$$
t_{i}^{1}(m)=\sum_{p=1}^{P} \sqrt{N} P d_{i, p}^{1} e^{j \pi / 4} e^{-j \pi \rho^{2} / N} \delta(p-m)
$$

where $\delta(p-m)$ denotes the delta function. Thus, from (40), we have arrived at the final result:

$$
\left|t_{i}^{1}(m)\right|=\operatorname{midd}_{i, m}^{1} \mid \sqrt{N} P
$$

which indicates that if $\left|d_{i, m}^{1}\right|$ is constant (MPSK), the transmit signal $\mathbf{t}_{i}^{1}$ has a constant envelope.

For the other users with $k=2,3, \ldots, K$, it is noteworthy that the same constant envelope result can be derived by referring to the procedures listed in (32)-(41).

\section{Acknowledgements}

This study was sponsored by the National Science Council, R.O.C., under the Contract NSC 100-2220-E-155-006. The authors would like to thank the Editor and the anonymous reviewers for their helpful comments and suggestions in improving the quality of this article.

\section{Competing interests}

The authors declare that they have no competing interests.

Received: 27 May 2011 Accepted: 27 October 2011

Published: 27 October 2011

\section{References}

1. HG Myung, J Lim, DJ Goodman, Single carrier FDMA for uplink wireless transmission. IEEE Veh Technol Mag. 1(3), 30-38 (2006)

2. F Adachi, $\mathrm{H}$ Tomeba, K Takeda, Frequency-domain equalization for broadband single-carrier multiple access. IEICE Trans Commun. E92-B(5), 1441-1456 (2009). doi:10.1587/transcom.E92.B.1441

3. T Frank, A Klein, E Costa, IFDMA: a scheme combining the advantage of OFDMA and CDMA. IEEE Wirel Commun. 14(3), 9-17 (2007)

4. HG Myung, DJ Goodman, Single Carrier FDMA: A New Air Interface for Long Term Evolution (Wiley, 2008)

5. 3GPP TS 36.211-Technical specification group radio access network; evolved universal terrestrial radio access (E-UTRA). Physical channels and modulation (Release 8)

6. F Horlin, A Bourdoux, E Lopez-Estraviz, L Van der Perre, Single-carrier fdma or cyclic-prefix CDMA with optimized spreading sequences? IEEE Trans Veh Technol. 57(5), 3230-3234 (2008)

7. S Hara, R Prasad, Design and performance of multicarrier CDMA system in frequency-selective Rayleigh fading channels. IEEE Trans Veh Technol. 48(5), 1584-1595 (1999). doi:10.1109/25.790535

8. R Van Nee, R Prasad, OFDM Wireless Multimedia Communications (Artech House, London, 2000)

9. K Adachi, M Nakagawa, Phase rotation/MC-CDMA for uplink transmission. Multi-carrier Syst Solution 41(VIII), 323-332 (2009)

10. JK Hwang, YL Chiu, RL Chung, A new class of MC-CDMA systems using cyclic-shift M-ary biorthogonal keying, in International Symposium on Intelligent Signal Processing and Communication Systems (ISPACS), Japan (December 2006)

11. JK Hwang, YL Chiu, A high-rate low-PAPR multicarrier spread spectrum system using cyclic shift orthogonal keying, in IEEE International Conference on Communications (May 2008)

12. CP Li, WC Huang, A constructive representation for the Fourier dual of the Zadoff-Chu sequences. IEEE Trans Inf Theory 53(11), 4221-4224 (2007)

13. S Beyme, C Leung, Efficient computation of DFT of Zadoff-Chu sequences Electron Lett. 45(9), 461-463 (2009). doi:10.1049/el.2009.3330

14. SH Han, JH Lee, An overview of peak-to-average power ratio reduction techniques for multicarrier transmission. IEEE Wirel Commun. 12(2), 56-65 (2005). doi:10.1109/MWC.2005.1421929 
15. F Giannetti, V Lottici, I Stupia, PAPR analytical characterization and reducedPAPR code allocation strategy for MC-CDMA transmissions. IEEE Trans Wirel Commun. 10(1), 219-227 (2011)

16. K Choi, K Kang, S Kim, Peak power reduction scheme based on subcarrier scrambling for MC-CDMA systems. IEE Proc Commun. 151(1), 39-43 (2004). doi:10.1049/ip-com:20040407

17. YS Cho, J Kim, WY Yang, CG Kang, MIMO-OFDM Wireless Communications with MATLAB (Wiley, 2010)

18. S Alamouti, A simple transmit diversity technique for wireless communications. IEEE J Sel Areas Commun. 16, 1451-1458 (1998). doi:10.1109/49.730453

19. DC Chu, Polyphase codes with good periodic correlation properties. IEEE Trans Inf Theory 18(4), 531-532 (1972). doi:10.1109/TIT.1972.1054840

20. Z Lin, P Xiao, B Vucetic, M Sellathurai, Analysis of receiver algorithms for LTE SC-FDMA based uplink MIMO systems. IEEE Trans Wirel Commun. 9(1), 60-65 (2010)

21. H Harada, R Prasad, Simulation and Software Radio for Mobile Communications (Artech-House, 2002)

22. JG Proakis, Digital Communications, 4th edn. (McGraw-Hill, 2001)

doi:10.1186/1687-1499-2011-144

Cite this article as: Deng and Hwang: Novel low-PAPR parallel FSOK transceiver design for MC-CDMA system over multipath fading channels. EURASIP Journal on Wireless Communications and Networking 2011 2011:144.

\section{Submit your manuscript to a SpringerOpen ${ }^{\circ}$ journal and benefit from:}

- Convenient online submission

- Rigorous peer review

- Immediate publication on acceptance

- Open access: articles freely available online

- High visibility within the field

- Retaining the copyright to your article

Submit your next manuscript at $\gg$ springeropen.com 